\title{
Studien über das Tapetum lucidum chorioideale.*
}

\author{
Von \\ Yuji Hosoya. \\ (細谷雄二) \\ (Aus dem II. physiologischen Institut der Lnirersität au Sendai, \\ Direktor: Prof. Dr. T. Fujita).
}

\section{Inhaltsverzeichnis.}

Einleitung.

Eigene Beobachtungen.

I. Strukturelle Beschaffenheiten des Tapetum lucidum und die Entstehungsweise der schönen schillernden Farbe desselben.

1. Versuchsmaterial und Herstellungsweise der Präparate.

2. Ausdehnung des Tapetums und Pigmentrerteilung in der Pigmentepithelschicht.

3. Verïnderungen des Farbenglanzes ron Tapetum durch chemische Einwirkungen.

4. Tapetumzellen und Tapetunfasern.

a. Herstellung der frischen mikroskopischen Prïparate.

b. Mikroskopicche Befunde am Sïugetiertapetum.

c. Mikroskopische Befunde am Selachiertapetum.

5. Doppelbrechung der Tapetunsubstanz.

II. Fluoreszenz des Tapetums.

1. Die Fluoreszenz des Tapetums und die der in Allali oder Sïure gelösten Tapetumsubstanz.

2. Herstellung eines Fluoreszenzschirmes aus der Tapetumschicht.

3. Absorptionsvermögen des Vollauges im Vltraviolett und die Fluoreszenz des Tapetums.

Zusammenfassung.

\section{Einleitung.}

Das bekannte Phänomen des Leuchtens der Hunde- und Katzenaugen im Dämmer, das auf dem Vorhandensein einer stark lichtbrechenden

* Der wesentliche Inhalt wurde in 4. (1925) und 5. (1926) japanischen Physiologenkongress mitgeteilt. Vgl. J. of Biophysics, 1927, 2, xxiI u. cxvil. 
Schicht "Tapetum lucidum" im Augenhintergrund beruht, hat zwar von alters her die Aufmerksamkeit der Forscher erregt, und man kann zahlreiche diesbezügliche Arbeiten in der Iiteratur auffinden. Was aber die strukturelle Beschaffenheit des Süugetiertapetums anbetrifft, sind die einzelnen Angaben mehr oder weniger von einander abweichend, und auch über die interessante Erscheinung von Fluoreszenz des Tapetums, die zuerst C. Hess ${ }^{1)}$ nachgewiesen hat, fehlt weitere Forschung. Betreffs der physiologischen Bedeutung des Tapetums blieb zwar noch bis jetzt die entscheidende Erklürung vorbehalten, aber es wird im allgemeinen als die Vorrichtung der Ausnutzung von geringeren Lichtreizen anerkannt, und, wie auch $\mathrm{Hess}^{2}$ angedeutet hat, handelt es sich bei der Fluoreszenz des Tapetums um eine Umwandlung von Strahlen von kürzerer für die Lichtempfindung. wirkungsloser Wellenlänge in solche von längerer, $\mathbf{d}$. h. in die beim Sehen im Dämmer wirksameren, z. B. vom Ultraviolett ins Blau- oder Gelbgrün. Also scheinen die spektralen Versuche der erregenden Strahlen und des erregten Fluoreszenzlichtes mir besonders interessant zu sein. Ich wurde durch diese Umstände zum eingehenden Versuche veranlasst und will hier die erhaltenen Resultate mitteilen.

\section{Eigene Beobachtungen.}

I. Strukturelle Beschaffenheiten des Tapetum lucidum und die Entstehungsweise der schönen schillernden Farbe desselben.

1. Versuchsmaterial und Herstellungsweise der Prïparate.

Das weit in verschiedenen Gattungen der Wirbeltiere verbreitete Tapetum lucidum lüsst in Säuger zwoi strukturell ganz verschiedene Arten unterscheiden : Tapetum cellulosum bei Carnivoren und Tapetum fibrosum bei Herbivoren. Auch unter den Fischen besitzen die Selachier das dem der Siiugetiere ähnliche Tepetum, und zwar das ans platten Zellen bestehende Tapetum cellulosum.

In dieser Untersuchung ist es mir zwar nötig, ein möglichst reichhaltiges Material sowohl nach Arten als auch nach Individualzahl an der Hand zu haben, aber an den Artenzahlen der Manmalien musste ich mich

1) C. Hess, Arch. f. vergl. Ophthalmol., 1911, II, 3.

2) C. Hess (1), 11 . 
wegen der leichten Zugünglichkeit mit gewöhnlichen I raussïlngetieren, d. h. Rindern, Pferden, Ziegen, Hunden und Katzen* begnïgen. Unter den Wasserberohnern licss idh das Seladhier (Cynias und Rajai) dic Tapetumbesitzenden vertreten.

Aus den lebensfrisch exstirpierten Angenbulbi stellte irly das Tapet umprïparat in folgender Weise her.

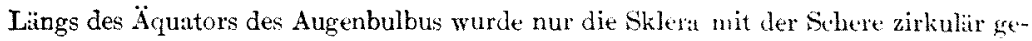
schnitten, wobei man den Bulbus nicht zu stark drücken darf, da sonst der Inhalt des Auges aus dem entstandenen Loch herausspritzt und weitere Behandlung dadurch erschwert wird. Die halbierte Sklera wurde nit feiner Pinzette nach hinten un den Sehnervenst:mm herum zurïckgeschlagen, (dabei wurde das Schillern der Tapezierung in der vitralen Fliche der Chorioidea, von hinten her betrachtet, mehr oder weniger angedeutet, besonders an der schwach pigmentierten Chorioidea war die Durchschimmerung sehr deutlich), und am Opticuseintritt abgeschnitten. Der Bulbus, der nun nur ron (horioidea und Retina umbüllt blieb, wurde iiquatorial halbiert, die hintere IIälfte in frisch angesammelten Kammerwasser abgewaschen, um sie rom enthaltenen Blut möglichst frei zu machen, und auf eine Objektglasplatte, die tapezierte (rordere) Fliiche nach oben, geklebt. Die Netzhaut wurde samt der Glaskörpermasse nit mit Kammerwasser befeuchteten Wattestiickchen entfernt, was leicht möglich ist, da die erstere fester der letzteren anhaftet als der L'nterlige.

\section{Ausdehnung des Tapetums und Pigmentrorteilmg in der Pigmentepithelschicht.}

Im Augenhintergrund bildet das Tapetum lucidum bei Süngetieren eine direkt oberhalb der Papilla nervi optici ausgebreitete, glinzende Fläche und hat die Gestalt eines ungleichseitigen Dreiecks, welches in der obereren Hälfte des Hintergrundes steht. Die Hypothenuse dieses Dreiecks bildet eine leicht gebogene Horizontallinie, die mit dem stïrksten Punkte der Krümmung die Papilla berührt und nur bei der Katze noch weiter unter die Papilla geht (vgl. Figg. 1, 2 und 3).

Was die Einzelheit der Ausdehnung und Form des Tapetums anlangt, stimmen die Befunde bei den Katzen, Iumlen, Rindern und Pferden im wesentlichen mit den Angaben von F. Briuk ke und M. Preusse überein, und beim Ziegentapetum sind dieselben ganz ähnlich wie beim Rinde. Bei Cynias und liaja ist der tapezierte Bezirk, wie V. Franz angegeben

*) Die Mehrzahl der Hunde- und Katzenmaterialien stammen ron den in dem I. physiologischen Institut andernzweclis gebrauchten Tieren. Herm Prof. Yasu. Sat alic, Direktor des Institutes, spreche ich dafiir meinen aufrichtigsten Dank aus.

3) E. Briicke, Arch. f. Anat. u. Physiol, 1845, 887.

4) M. Preusse, Arch. f. wiss. u. prakt. Tierheilk., 1882, 8, 264.

5) T. Franz, Jenaische Zeitschr. f. Naturwis, 1905, 40, 697 . 
hat, ausgedehnter als bei den Haussäugetieren und nimmt fast den ganzen Augengrund ein.

Die Pigmentepithelschicht der Netzhaut besteht bei allen von mir untersuchten Säugetierarten aus einer einfachen Lage sechseckiger platter Zellen, und die Pigmententwicklung der einzelnen Teile ist in ein und derselben Netzhaut sehr verschierlen. Im nicht tapezierten Gebiete ist das Epithel zwar stark pigmenthaltig, aber das an den Rändern der tapezierten Partie anliegende zeigt sich mehr oder minder pigmentąrm. Im mittleren Bereiche des $\mathrm{Ta}$ petums sind die Epithelzellen vollständig pigmentfrei (abgesehen vom Pferde), und nach den Rändern zu gehen sie allmählich in die schwarz pigmentierten Epithelien über.

Nur beim Pferdeauge ist das Epithel auch im tapezierten Teile nicht pigmentfrei, sondern sogar in der Mitte dieses Gebietes wenn auch sehr schwach pigmentiert. Beim Selachier (Cynias oder Raja) ist, wie 
Franz $z^{6)}$ angegeben hat, das Aussenblatt der Netzhaut völlig unpigmentiertes einschichtiges Epithel, soweit es der Pars optica retinae anliegt.

3. Veründerungen des Farbenglanzes von Tapetum durch chemische Einwirkungen.

Beim Rindertapetum wurde von $\mathrm{Hess}^{i)}$ bestätigt, dass der schön irisierende blaugrüne Farbenglanz des Tapetums beim Trocknen verschwindet und nicht vom übrigen Chorioideateil unterscheidbar wird. Auch bei Pferde- und Katzentapetum konnte ich dieselbe Erscheinung nachweisen, während das Tapetum beim Hunde nach dem Austrocknen grauweisslich (mehr oder minder kreidig weiss) und noch deutlich erkennbar blieb (Fig. 2). Mit dem Einbringen ausgetrockneter Präparate ins Wasser oder in die Ringerlösung tritt die verlorene Farbe sogleich wieder auf, doch hat diese an Glanz ziemlich eingebüsst und zeigt meist einen tiefer bläulichen Ton.

In verschiedenen Säuren, wie Salz-, Schwefel-, Salpeter- und Essigsäure in einer Konzentration von etwa $5-10 \%$, behält das R. L.

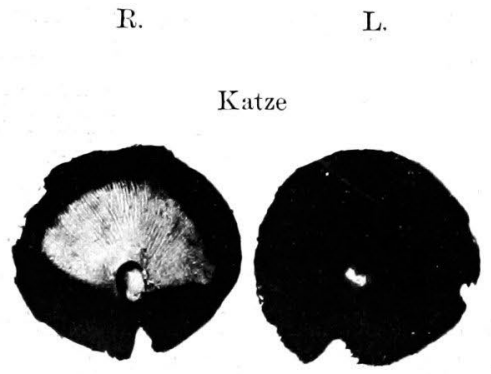

Hund

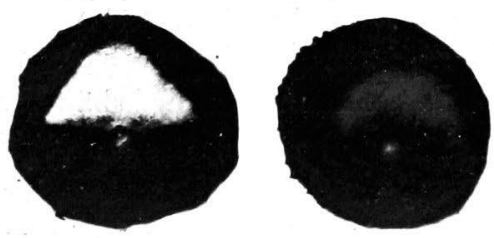

Fig. 2.

Frische Tapeta : rechts $(\mathbf{R})$, befeuchtet und links (L), getrocknet.

Rindertapetum seine Farbe bei (Hess), allerdings nach einmaligem Verschwinden und immerhin in dunklerer Nuance; in 10-25\% Alkalien wird sie dagegen allmählich grauweisslich und glanzlos. Beim Pferdetapetum ist das Verhältnis etwas anders, und zwar tritt beim Zusatz von Alkalien nach einmaligem Verschwinden eine glïnzcnde dunkelgrüne Farbe auf.

6) V. Franz (5), 769.

7) C. Hess (1), 5 . 
Katzen- und Hundetapetum verlieren ihre Farbe sowohl in Süuren als auch in Alkalien gänzlich. Das Katzentapetum ist aber widerstandsfähiger gegen solche Agention als das Hundetapetum. In $5 \% \mathrm{HCl}$ geht die Farbe des frischen Tapetums des Hundes nach einigen Stunden zu grunde, das der Katze aber bleibt einige Tage lang fast unveründert, und in 10\% Konzentration verschwindet die Farbe beim Hunde binnen ca. 2-3 Minuten, bei der Katze erst im Laufe mehrerer Stunden. Beim Aufgiessen von Alkalien (5, 10 und 25\% $\mathrm{KOH}$ oder $\mathrm{NaOH}$ ) erfolgt das Verschwinden der Tapetumfarbe ebenso beim Hunde wie bei der Katze sehr rasch, z. B. behült das 'Tapetum die Farbe der beiden in 10\% $\mathbf{K O H}$ nur einige Minuten lang bei. Nach dem Austrocknen nimmt die Resistenz der Tapetumfarbe gegen diese Chemikalien stark zu, also widersteht die Farbe des ausgetrockneten Präparats aus der Katze der dauernden Einwirkung der 25\% $\mathrm{HCl}$ oder $\mathrm{KOH}$ einige Stunden lang, um sich dann allmählich zu verlieren. Immerhin ist solches Präparat des Hundes gegen die oben genannten Agentien viel schwächer resistent und verliert die Farbe innerhalb einiger Stunden vollständig. Die so behandelten Präparate gewinnen nach dem Austrocknen auch in Wasser oder Ringerlösung ihre einmal verlorene Farbe nicht mehr wieder.

In $3-5 \%$ Formol zeigt die Veränderung des Katzen- und Hundetapetums einen wesentlichen Unterschied; und zwar behält das Tapetum der Katze, gleich wie das des Rindes und des Pferdes, während des längeren Liegens in Formol seine cigentümliche Farbe fast unveründert bei, beim Hunde dagegen wird das im Formol liegende Präparat ganz allmählich grauweiss und glanzlos, und verliert sich endlich, etwa im Laufe von 5-6 Tagen (Fig. 3). Diese Erscheinung des Hundetapetums geht mit einer strukturellen Veränderung einher, worauf ich
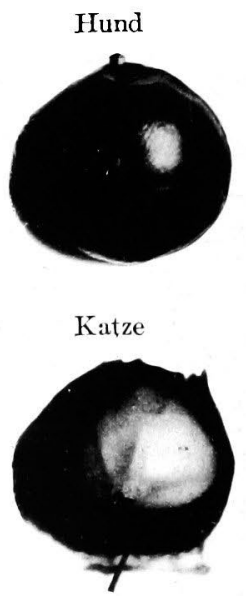

Fig. 3.

Nach 5 tïgiger Auf bewahrung in 5\%ó Fornol: Das Tapetum ist beim Hunde verloren gegangen, aber bei der Katze gut erhalten. im nächsten Kapitel noch eingehender zurücklkommen werde.

Das Verschwinden der Tapetumfarbe beim Austrocknen oder Aufträufeln von Alkalien kann man, wie $\mathrm{Hess}^{8)}$ angab, dadurch erklären, 
dass die Tapetumschicht durch diesen Prozess durchsichtign wird und daher die dahinter gelegene Pigmentschicht durchschimmert. Aber die z. B. beim Befeuchten der ausgetrockneten Prïparate oder Zusatz von Säure wiedergewonnene Farbe ist nienals gleich wie die ursprüngliche, sondern immer von tiefer bläulichem Ton, und der Glanz wird ziemlich stark beeinträchtigt. Dies beruht darauf, dass durch die oben erwähnte chemische Einwirkung die Tapetumschicht mehr oder weniger getrübt wird. Solche Trübung tritt, wie im nächsten Kapitel angef ührt wird, sowohl im I aufe der mikroskopischen Beobachtung der dünnen Tapetumschicht als auch beim ungenügend entwickelten Tapetum des Jungen von Hund, Katze und des Fötus von Rind dentlich auf.

Beim Selachier ist die Farbe des Tapetum cellulosum herrlich glinzend gelbgrün, und mit der Änderung des Lichtreflexionswinkels zeigt die tapezierte Fläche gleich wie die Perlmuttcr ein schönes Farbenspiel. Läisst man das Tapetum trocknen, so wird es tief schwarz, aber in passender Neigung zeigt es dunklen Silberglanz. Nach dem Eintauchen des ausgetrockneten Präparates in Scewasser kehrt nicmals die ursprüngliche Farbe wieder. Wird es in frischem Zustand in 20\%5 Salzsinre gebracht, so erscheint es nach einigen Minuten tiefschwarz, aber gegen Alkalien, z. B. $100_{0}^{\circ} \mathrm{KOH}$, hat es ziemlich starke Resistenz; in konzentrierten Alkalien allerdings verschwindet der Farbenglanz sofort. Nach dem Austrocknen geht die ursprünglich schillernde Farbe verloren, indem die charakteristische Anordnung einzelner in den Tapetumzellen eingeschlossenen Kristalle, wie unten zu erörtern ist, infolge der Schrumpfung der Tapetzellmembranen gestört wird.

\section{Tapetumzellen und Tapetumfascrn.}

\section{a. Herstellung der frischen mikroskopischen Präparate.}

Am Eingange dieses Versuches nahm ich die mikroskopisthen Untersuchungen des Tapetums mit den Schnittprïparaten des fixierten Materials vor; jedoch merkte ich nach einigen Fixationsversuchen, dass sich die Farbe desselben durch das Fixationsverfahren mehr oder minder veründert. Besonders beim Ifunde wird der schöne Farbcnglanz nicht nur durch dic Fixation, sondern auch beim Erfrieren, wie es bei der Herstellung des Gefricrschnittes der Fall ist, merklich becintrüchtigt ; die blaugrüne oder gelbgrüne irisierende Farbe des frischen Tapetums wird danach ganz matt kreidig weiss. So nahm ich immer die folgende Ablösungsmethode mit dem 
lebensfrischen Material vor.

Zuerst wurde die Sklera in der schon besprochenen Weise weggenommen. Dann wird die Chorioidenschicht mit einer feinen Pinzette an der Opticuseintrittsstelle gefasst und mit leichtem Zug von der Tapetumschicht abgelöst. Da in der Gegend des Sehnerveneintritts die Zellschicht des Tapetums am müchtigsten ist, nach den Ründern zu immer dünner und am äussersten Rande nur ein- oder zweischichtig wird, so konnte ich aus diesen Randteilen Stückchen der genügend dünnen Tapetumschicht bekommen, die zu mikroskopischen Studien ganz geeignet sind. Solche Stückchen wurden in Kammerwasser auf Objektglas gebracht, mit Deckglas bedeckt und mikroskopiert. In gleicher Weise konnte ich auch das Fibrcsumpräparat sehr leicht herstellen.

\section{b. Mikroskopische Befunde am Säugetiertapetum.}

Tapetum cellulosum besteht bei Hunden und Katzen aus grossen platten Zellen, die ca. 25-60 $\mu$ lang, 12-25 $\mu$ breit und unregelmüssig sechseckig sind. Nach O. Zietzschmann $n^{9)}$ wären die Tapetumzellen des Hundes kleiner als die der Katze, jedoch ergaben meine zahlreichen Messungen sehr schwankende Zahlen, und auch im Mittel konnte ich keinen einheitlichen Grössenunterschicd zwischen beiden bestätigen.

In den Katzentapetumzellen, besonders beim Gebrauche der Ölimmersion, kann man sich leicht davon überzengen, dass der ganze Zelleib mit nadelförmigen kristallähnlichen feinen ca. 0,4-0,6 $\mu$ dicken Gebilden erfüllt ist (vgl. Fig. 4). Solche Zelleinschlüsse kommen nach Zietzschmann $n^{103}$ nur bei der Katze vor, aber nicht beim Hunde. Bei Brücke ${ }^{(1)}$ und M. Schultze, ${ }^{12}$ die auch das Hundetapetum untersuchten, befindet sich keine nähere Beschreibung über seine Zellstruktur.

Ich konnte immer gleiche Gebilde auch in den Hundetapetumzellen nachweisen, soweit diese von ganz frischem Material stammten.

Dass das Protoplasma der Hundetapetumzellen eine deutliche feine

9) O.Zietzschmann, in W. Ellenbergers IIandb. d. vergl. mikros. Anat. d. Haustiere, Bd. 1, 1906, 439.

10) O. Zietzschmann (9), 440.

11) E. Brücke (3), 396-398.

12) M.Schultze, Sitzungsber. ii.d. niederrhein. Gesellsch. f. Natur- u. Heilk. (Nachtrag z. Pericht ii. d. Sitzung vom 27. Novera. 1871, gehalten in d. Verhandl. d. naturhist. Ver. d. preuss. Rheinlande u. Westfalens.) Jg. 29, 1872, 210. 

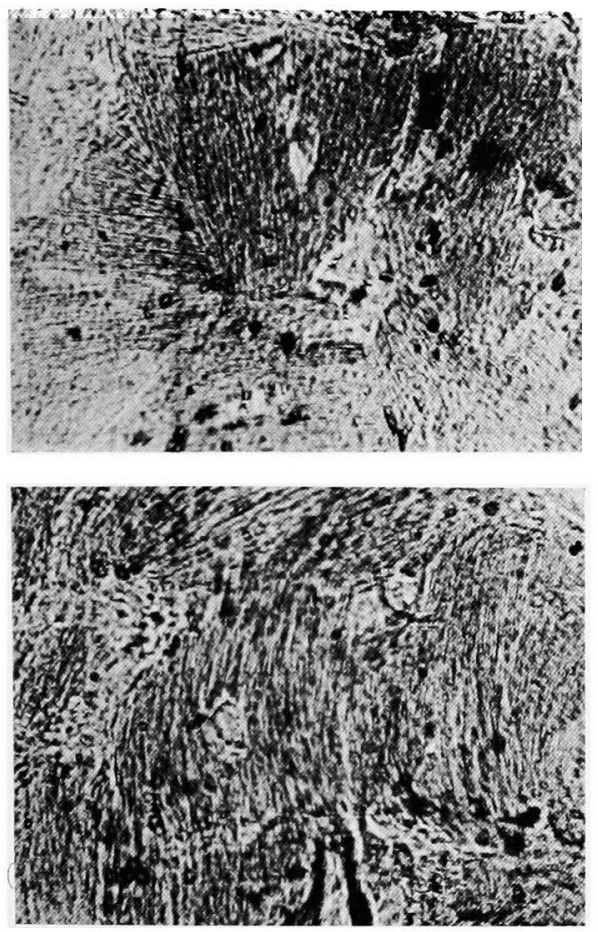

Fig. 4.

Frische Tapetumzellen (im frischen Kammerwasser abpräpariert) des Hundes (1) und der Katze (2). Zeiss: Obj. $90(1,25)$, Okul. $10 \times$, Leitz Makam.
Längsstreifung zeigt, wurde schon in 1882 von Preuss $\mathrm{e}^{13)}$ beschrieben und in ; Histologie der Haussäugetiere " von W. El(1) lenberger ${ }^{14)} 1887$ Wort für Wort zitiert. Zietzschmann ist aber anderer Ansicht, deun er beschreibt mit folgenden Worten : „im Tapetum des Hundes finden sich Zellen ganz anderer Struktur (als die in dem der Katze). Ihr helleres Protoplasma zeigt nur leichte wolkige Trübungen (Fig. 337); sie sind auch kleiner als die der Katze (Fig. 338) usw."**

Die kristallähnlichen Gebilde sind bei ein und derselben Zelle miteinander fast parallel gereiht. Bei der Katze und beim Hunde finden wir sie in ähnlicher Anordnung, aber in etwas verschiedener Form; nadelförmig bei der Katze, mehr stäbchenförmig beim Hunde. Jedenfalls ist ihre Dicke bei beiden von gleicher Feinheit, die zur Erzeugung der Lichtinterferenz genügend ist (ca. 0,4-0,6 $\mu$ ).

Bezüglich der Zellstruktur des Katzentapetums fanden Preusse und auch Zietzschmann, dass die Zellen ,eine ganz eigentümliche Streifung, welche parallel den beiden kurzen Seiten des Sechseckes verläuft, zeigt. Jeder einzelne durch diese Streifung hergestellte Abschnitt zeigt wiederum

13) M. Preusse (4), 279.

14) W. Ellen berger, Handb. d. vergl. Hist. u. Physiol. d. Maussïugetiere. Bd. 1 (Vergl. Hist. d. Haussïugetiere), 1887, 591.

*) Vgl. auch Ellenberger u. Schumacher: Histologie der Hanssïngetiere. V. Aufl., 1914, 311 u. Landois-Rosemann: Lehrbuch d. Physiol., 18. Aufl., 1923, 760. 
eine feine Streifung in der Querrichtung (Figg. 4 u. 5), etc." Dieses Bild habe ich auch manchmal gesehen, wenn sich die Präparate in beginnender Mazeration befanden oder von irgend einem Fixationsmittel angegriffen wurden (Fig. 5), aber in der Anordnung der Einschlüsse kann man, so lange die Zellen ganz frisch sind, die von Katze und Hund nicht unterscheiden. Ferner sagte Preusse, ${ }^{15)}$ dass er nicht bei allen Katzentapetumzellen einen Kern nachweisen könnte und erst nach dem Verschwinden (in Essigsïure) der mikrokristallähnlichen Gebilde die Kerne deutlich hervorträten. Bei meiner Untersuchung mit ganz frischem Präparat sind die Kerne dagegen meist in der Mitte der Zelleinschlüsse gut nachweisbar.

Beim Hunde sind diese Zelleinschlüisse, im Gegensatz zu denen der Katze, gegen die Einwirkung von Säuren und Alkalien, auch von ganz verdünnter $L_{\jmath}$ sung $(0,5 \%)$, sehr schwach resistent, so verschwinden

15) M. Preusse (4), 279.

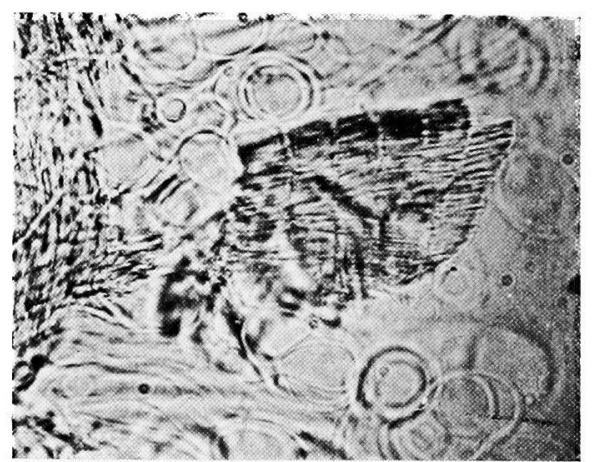

Fig. 5.

Katzentapetumzellen : in 5\% Formol 24 Stdn. lang aufbewahrt. Zeiss : Obj. 90 $(1,25)$, Okul. $10 \times$, Leit z Makam.
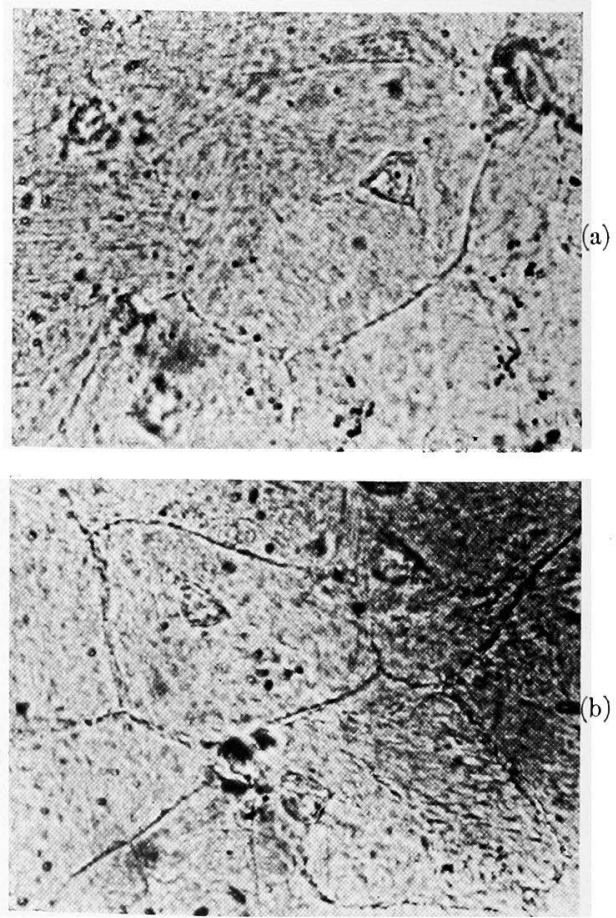

Fig. 6 .

Hundetapetumzellen : in $5 \%$ Formol 24 Stdn. (a) resp. 48 Stdn. (b) lang aufbewahrt. Zeiss: Obj. $90(1,25)$, Okul. $10 \times$, Le it z Makam. 
sie bei Behandlung mit den Fixier-(Aufbewahr-) flüssigkeiten, wie Forma$\operatorname{lin}(3-5 \%$ ) oder Sublimatlösung (Sublimat-Essigsäure oder Sublimat-Kochsalz), schon in wenigen Stunden aus dem Gesichtsfeld, wie man sich leicht davon überzeugen kann. Ihr Torkommen im Hundetapetum war bisher vielleicht deshalb übersehen, weil sie gewöhnlich in fixierten Priiparatei untersucht wurden (vgl. Fig. 6). Bei Anfbewahrung im Kammerwasser ist die Zellstruktur sehr lange, ja sogar fünf oder sechs Tage lang haltbar.

Tapetum fibrosum bei Pferden, Rindern und Ziegen besteht aus wellig parallel verlaufenden Fibrillen, die ganz ähnlich wie die feinen Streifen in der Innenfläche von Perlmutter cin schöncs Farbenspiel mit der Änlerung des Einfallswink ls von Licht zeigen, und ron beinahe gleicher Feinheit $(0,4-0,6 \mu)$ wie die mikrokristallähnlichen Gebilde des Tapetum cellulosum sind. Aber die Anwesenheit doppelbrechender Mikrokristalle in den Tapetumfasem konnte ich nicht nachneisen. Dieser letzte Befund stimmt mit dem von Hess, ${ }^{16}$ aber nicht mit dem von $A$. Pätter ${ }^{15}$ überein.

Da das Tapetum cellulosum sowohl bei Katze als auch bei Hund in seinen Zellen feine Büschel von nadel-oder stäbchenförmigen Gebilden von so kleiner Dicke, dass sie zur Erzeugung der Lichtinterferenz genïgend fein sind, enthält-obgleich dieses nicht so dentlich den Farbenwechsel wie Fibrosum zeigt, vielleicht weil die Nadelbüschel nicht immer parallel wic Fibrosumfasern angeordnct sind-so liegt es nahe, den Farbenglanz des Säugetiertapetums (in beiden Arten, d. h. sowohl im T. fibrosum und im T. cellulosum) als das Interferenzphänomen durch die Gitterbeugung anzunehmen. Diese Ansicht scheint noch stïrker in der 'Tatsache begründet zu sein, dass das Tapetum ncugeborener Tiere, bei dem die Zelleinschlüsse noch amorph sind, keine Irideszenz wie das der ausgewachsenen zeigt.

Schultze ${ }^{18)}$ fand nämlich beim Katzentapetum, dass die Kritalle zwei Wochen nach der Geburt noch gïnzlich fehlen, uml in dieser Zeit anch von Metallglanz in der Chorioidea noch keine Spur vorhanden ist.

Ich habe gefunden, dass nicht nur bei den Neugeborenen von Katzen, sondern auch von Hunden die kristallähnlichen Zelleins hlüisse noch nicht erkennbar sind, und der Zelleib mehr oder weniger getrübt ist. Das Tapetum beginnt erst mit der vollen Entwicklung der kristallähulichen Zelleinschlüsse (2. Lebensmonat bei Katze und Hund) seinen dharakteristischen Farbenglanz zu zeigen.

16) C. IItess (1), 4.

17) A. Piater, Organologie des Auges in Graefe-Saem ischs IIandb. d, geamt. Augenheilk., III. Aufl, 1912, 274.

15) M. Schultze (12), 211. 
Beim Rinderfötus ist die Tapetumfarbe intrauterin schon hervorgetreten, wenn auch nicht ganz ähnlich wie beim Erwachsenen, sondern mehr zum Gelb genejgt. Neuerdings wurde ron E. M u r r ${ }^{19)}$ die Entwicklung des T. fibrosum bei Schaf und Rind ausfuhrlich studiert, und es ergab sich dass die Irideszenz des Rindertapetums erst nach der Verfeinerung von Tapetumfasern (nach 5. Fötalmonat) auftritt. Dieses Resultat stimmt mit meiner Erfahrung mit zwei Rinderföten, ron denen einer 3 und der andere 10 Monate alt war, gut iberein; es zeigte sich nänlich, dass beim ersteren die charakteristische Tapetumfarbe noch nicht entwickelt, sondern nur grauweislich, aber beim letzteren gelbgrün gut schillernd war.

Bein Tapetum erwachsener Ticre kommt die matt blaue Farbe manchmal in Butracht, wenn die Zellen ihre cigentïmlich streifige Struktur verlieren, wie z. B. bei Erfricren, Fixieren oder Veralten, und die blane Färbung stellt nichts anders dar als die Farbe trüber Medien vor eincm schwarzen Hintergrund (Chorioidea). In den Tapetumzellen der mit Formol oder Sublimat fixicrten Schnittpriiparate, wie Zietzsehman $n^{n}$ beschrieben hat, sind freilich die kristallïhnlichen Gobilde schon verloren gegangen.

Die frisch abgeschïlte dïnne Membran des Sïugeticrtapetums zeigt unter dem Mikroskop in auffallendem (reflektiertem) Lidite eine bunte schillernte Farbe; bei der Zellulosummembran (Katze und Hund) herrscht die glänzend gelbe und yrüne Farbe vor, abor bei der Fibrosummembran (Rind, Pford odcr Ziegre) ist diese Verfärbung so viclfach, dass den wellenartig gewundenen Fibrillen entlangr tist alle Spektralfirben wahrgenommen werden. Wenn nan dic Membran des Fibrosums oder des Cellulosums mit durchfallendem Lichte beleuchtet, so sicht man leicht, dass alle Teile derselben ihre Farben ändern und zur im auffallenden Lichte ersehienenen Farbe komplementiir verfäbt werlen. Mit dem Veralten des Präparats verindert sich die Farbe des Tapetums, doch bleibt sie in durchfallenden und in auffallendem Lichte stets komplementiir ; z. B. wird nach stundenlanger Aufbewahrung in Ringerlisung das der Katze oder des Hundes in auffallendem Lichte ungesittigt indigoblau, und zeigt dabei in durchfallendem Lichte gelbliche Farbe. Wenn auch die Farbe der Fibrosummembran in Ringerlösung in noch lïngerer Stundenanzahl keine Verïnderung aufweist, so tritt eine solche dich im Laufe von mehreren Tagen auf.

\section{c. Mikroskopische Befunde am Selachiertapetum.}

Wemn der Angenhintergrund von Selachier (Cynias und Raja) bei

19) E. Murr, Zeitschr. f. wiss. Biol., Abt. F, Zeitsehr. f. Zellforsch. u. mikros. Anat., 1925, 2, 701. Bein neueren Versuch iiber die Entwicklung des IIauskatzentapetums (rgl. dieselbe Zeitschr., 1927, 6, 315) hat er bestaitigt, dass der Metallglanz des Tapetums in 7 . Lebensworhe entsteht.

20) O. Zietzschmann $(9), 489$. 
schwacher Vergrösserung mit auffallendem Licht mikroskopiert wird, so zeigt die tapezierte Fläche einen etras anderen Befund als die des Süngetiertapetums. Die glänzende Oberfläche sieht durch die zahlreichen schwarzen Pigmentzellfortsätze, die von der Chorioidea durchtreten, umregclmässig wellig geschlängelt aus, und dazwischen sind Reihen von Tapetumzellen irisierend eingelagert. ${ }^{.1}$ Aber in der etwa $1 \mathrm{~cm}$ langen schmalen Zone, die sich einige mm oberhalb der Papilla horizontal hinzieht, sind die Pigmentstreifen sehr schwach entwickelt oder fehlen gïnzlich. Gerade beim Sclachier entspricht diese Zone der streifenförmigen Area, die als die Region des schürfsten Sehens bezeichnet wird.

Beim Selachier scheinen die Kristalle in der Tapetumzellschicht ganz locker enthalten zu sein, da min unter dem Mikroskop deutlich bemerkt, dass sie beim leichten P'ressen des mit Seewasser eingebetteten Präparats mit dem Deckglase die eigentliche Zellschicht verlassen und frei schwimmend werden. Sie sind unter $1 \mu$ dick, ca. $5 /$ breit, $10 \%$ lang und stellen im grossen und ganzen rhombische Täfelchen dar. Im auffillenden Licht zeigen diese einen schönen, blaugrün schillernden Glanz und verschwinden bisweilen durch Molekularbewegung für Augenblicke ans dem Gesichtsfeld, erscheinen dann wieder, indem sie, durch reflektierendes Licht in dunklerem Gesichtsfeld flimmernd, allmühlich die Stelle veründern. Im durchfallenden Licht sind die Kristalle durchsichtig und farblos. Wraden die frisch vom Tapetum isolierten Kristalle unter den Mikroskop in 50\% $\mathrm{HCl}$ oder Formol eingetaucht, so verlieren sie allmählich die schn̈ne Irideszenz und werden zu schmutzig schwarzen Trümmern.

Beim Selachier ist es sehr wahrscheinlich, dass das schöne Schillern nach dem Prinzip von Lichtinterfercnz an dünnen Schichten entsteht, da die Tapetumzellen, wie oben erwähnt, mit sehr dünnen durchsichtigen Täfelchen gefüllt sind.

Bein Eingiessen der Salzsiure löst sich die Tapetumsubstanz, und zwar noch $P$ iit te ${ }^{n-9}$, unter Gasbildung $\left(\mathrm{CO}_{2}\right)$, ich konnte jedoch solche (awentwicklung, wie auch $\mathrm{H}_{\text {ess, }}{ }^{23}$ ) weder makroskopisch noch mikroskopisch wahrnehmen.

Die Prifung auf Guaninreaktion nach $\mathrm{Capranica}{ }^{24}$ ) welche der Mikrokristalle ron Guaninkalk zugrunde liegt, gelang mit der Tapetumsubstanz der Säugetiere nicht, wohl aber mit den Kristallen des Selachiertapetums (E. Berger), ${ }^{25}$ was die Verschiedenheit der cheuischen Natur der beiden Arten des Tapetums (ron Siugern und ron Selachier) beweist.

21) V. Franz (5), 769-770.

22) A. Pitter, Organologie des Auges in Graefe-Saemischs Handb. d. gesant. Augenheilk., II. Aufl., 1908, 256.

23) C. Hess (1), 4 .

24) S. Capranica, Zeitschr. f. physiol. Chem., 1880, 4, 233.

25) E. Berger, Morphol. Jahrb., 18s:, 8, 97. 


\section{Doppelbrechung der Tapetumsubstanz.}

Wie die meisten organischen Substanzen doppelbrechende Eigenschaft besitzen, ${ }^{26)}$ so kommt diese anch den kristallïhnlichen Gebilden des Süugetiertapetums zu.

Ich wandte zu der Untersuchung dieser Eigenschaft einen Polarisationsapparat und ein Mikroskop mit drehbarem Objekttisch (Zeiss, Stativ $\mathrm{IPh}_{\mathrm{C}}$ 10) an. Bei der Beobachtung wurde jedes störende Nebenlicht durch cinen den Objekttisch umgebenden Schirm aus schwarzem Karton abgehalten.

An den Mikrokristallen des Selachiers konnte ich die Doppelbrechung nicht konstaticren. Schult $\% \mathrm{e}^{27}$ beobachtete, dass die durch Zerzupfung isolierten Kristalle des Tapetum cellulosum der Sängetiere Doppelbrechung zeigen. Auch ich habe gefunden, dass sowohl die isolierten kristallähnlichen Gebilde des Cellulosums als anch die zerzupften Tapetumfasern schwach doppelbrechend sind. Bei den nicht zerzupften Tapetumzellen und -fasern tritt dicse Erscheinung deutlicher auf, und noch stärker wurde dieselbe von her dünnen Schicht des Cellulosums und Fibrosums gezeigt.

Wemn die beiden Polarisationselenen parallel stehen, so erscheint die Zellulosums hicht der Katze oder des Hundes gleich mässig hell, und je mehr sich die Drehung des Analysators der rechtwinkligen Kreuzung nähert, desto dunkler wird das Gesichtsfeld, in welchem aber hier und dort helle kleine Felder glänzend geblieben sind. Dieschellen kleinen Felder scheinen auf den ersten Blick den einzelnen Tapetumzellen zu entsprechen, jedoch zeigen sie durchaus nicht die einheitliche Form und Grösse derselben. Wenn man das Bild irgend eines dieser Hellfelder zuerst in hellem und damn in dunklem Gesichtsfeld genauer betrachtet, so crkennt man leicht, dass bei letzterem immer ein bestimmter Protoplasmateil, in welchem die nadelförmigen Zolleinschlüsse in gleicher Richtung nebeneinander gereiht sind, hell erscheinen und der übrige Teil des Zelleibs ganz dunkel. Die einzelnen hellen Particn im Gesichtsfelde stehen miteinander ungefïhr parallel oder annühernd in einem Winkel von 45 Grad.

Um die Abwedsselung von Holl und Dunkel in den Tapetumzellen etwas näher zu betrachten, wurde der Objekttisch von eimer dunklen Stellung ausgehend um die Axe des Polarisationsmikroskops nach und nach 360 Grad gedrelit, wobei mit jeder 45 Grad Drehung die helle Partie dunkel und die dunkle hell wurde.

26) K. v. Erlach, Arch. f. Anat. u. Physiol., 1847, 31:3.

27) M. Sehultze (12), 211 . 
Ebenso wie die Zellulosum- zeigt die Fibrosumschicht doppelbrechende Eigenschaft, und es ist sehr merkwürdig, dass sie beim Drehen des Analysators sog. „Pleochroismus“ zeigt. Das bei hellem Gesichtsfeld sichtbare Rot verändert sich im Laufe der Drehung des Analysators, und wenn die Drehung den 90 Grad erreicht, tritt die Komplementärfarbe desselben(Blaugrün) auf.

Da die Schicht des Cellulosums diese optische Eigenschaft nach dem Verschwinden der Zelleinschlüsse nicht mehr beibehült, und gleichzeitig damit auch die Schillerfarbe derselben zugrunde geht, kann man die Vermutung aussprechen, dass sie bei der Entstehung der eigentümlichen Tapetumfarbe eine Rolle spielt.

Die Mikrokristalle des Selachiertapetums zeigen keine Doppelbrechung.

Es scheint, wie K. v. $\mathrm{Erla}^{2{ }^{28}}$ und Helm hol $\mathrm{z}^{29}$ hervorgehoben haben, diese Doppelbrechung, welche besonders bei ptlanzlichen Membranen und Fasern auffallend ist, eine allgemeine optische Eigenschaft der organischen Substanzen zu sein.*

\section{Fluoreszenz des Tapetums.}

Es war Hess, ${ }^{30)}$ der zuerst das Vorhandensein der Fluoreszenz am Tapetum der Säugetiere mit dem Blau-Uviolglas und dem elektrischen Bogenlichte bemerkte, aber diese interessante Erscheinung ist seither noch recht vernachlässigt worden. Diese optische Eigenschaft ist, wie eingangs erwähnt, für das Tier, welches in der Dümmerung tätig ist, als ein sehr wichtiges Hilfsmittel zum Sehen durch Ausnutzung geringer Lichtmengen zu betrachten. So habe ich die Fluoreszenz des Tapetums spektroskopisch und spektrographisch eingehender untersucht.**

Bei den Versuchen muss man darauf achtgeben, dass die mässige Fluoreszenz des zu prüfenden Materials anch durch geringe Beimengung der sichtbaren Strahlen des im Versuchsraum befindlichen Nebenlichtes in hohem Masse verdeckt wird, und dass man diese daher am bequemsten im Dunkelzimmer anzustellen hat. Als Lichtquelle, die bei genügender Intensität nur ultraviolettes Licht gibt, habe ich einen H. Leh m an nschen ${ }^{31)}$

28) K. จ. Erlach (26), 326 .

29) H. v. Helm hol t z, Handb. d. physiol. Optik, 1896, II. Aufl, 571.

*) Es sei nebenbei bemerkt, dass für die Entstehung der schönen Interferenzfarben an gittrigen Systemen von lebenden Organismen z. B. Perlmutter, Federn der Insekten oder Vögel und Hautschuppen der Schlangen u. s. w. die schon bestätigten Annahmen über die Rolle der Gitterstruktur gelten, dass aber sehr interessanterweise die Hautschuppen der Schlange, wie H. Nagaoka und T. Futaga mi (Bulletin of the Institute of Physical and Chemical Research, 1924, 6, 914. Jap.) neuerdings mitteilte, nicht nur gitterförmige feine Streifung, sondern auch doppelbrechende Eigenschaft zeigen.

30) C. Hess (1), 10.

**) Hier drücke ich Herrn Dr. J. Oku ubo, Professor der Physik der hiesigen Universität, für seine wertvollen Ratschläge über die spektrographischen Untersuchungen meinen herzlichsten Dank aus.

31) H. Lehmann, Verhandl. d. Deutsch. Phys. Ges., 1910, 12, 890. 
Ultraviolettstrahlenfilter (U-V-Filter), der vor einer Nickelbogenlampe gesetzt wird, benutzt; die eine der Doppelküvette des Apparats, deren Wand aus Jenaer Blau-Uviolglas, das die Strahlen von 4500_3000 A. E. Wellenlänge und das Rot durchlässt, besteht, ist mit verdünnter wässeriger Iösung von Nitrosodimethylanilin, welches für Strahlen von 4000—2800 A. E. Wellenlänge durchlässig ist, und die andere mit $20 \%$ Kupfersulfatlösung, welche das Rot zurückhält, zu füllen. Die gefüllte Küvette hat also die Eigenschaft, nur ultraviolettes Licht aus dem Spektralgebiet von 3000-4000 A. E. durchzulassen, und die durchgelassenen Strahlen sind von grosser Reinheit (Fig. 7). Zu photographischen Versuchen brauchte ich ein Quarzspektrograph E. 37 von A. Hilger und panchromatische Platten von Ilford.

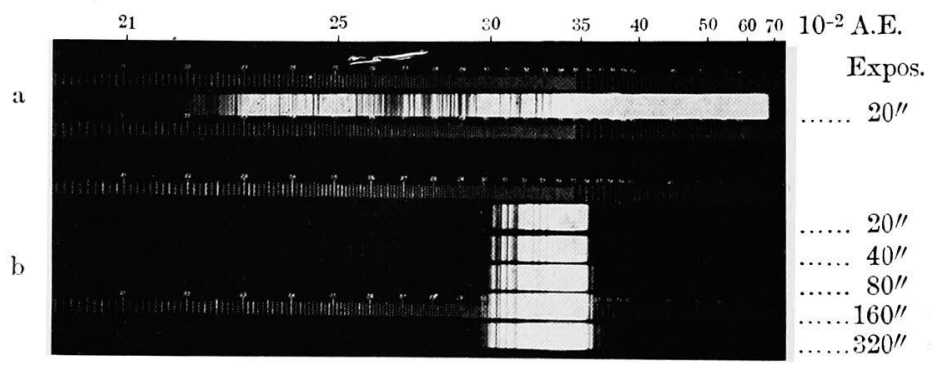

Fig. 7 .

a. Nickelbogenlicht. b. Durch den U-V-Filter passiertes Licht (bei verschiedener Expositionsdauer. Kollimatorspalte : 0,046 mm breit).

1. Die Fluoreszenz des Tapetums und die der in Alkali oder Sïure gelösten Tapetumsubstanz.

Die Fluoreszenz des Tapetums kann man in folgender Weise sehr leicht nachweisen : Lässt man auf einer tapezierten Chorioideaschicht, gleichgültig ob in frischem (z. B. die hintere Ḧ̈lfte des frisch enukleierten Auges) oder in veraltetem Zustand, das Licht der gewöhnlichen Bogenlampe (Kohlenelektroden) durch cin Blau-Uviolglas fallen, so leuchtet das tapezierte Gebiet in einem schönen gelbgrünen Lichte. Die Intensität derselben ist am stärksten bei Katzen-, schwächer bei Hunde- und am schwächsten bei Pferde-, Rinder- und Ziegentapetum. Beleuchtet man dagegen durch gelbes oder rotes Glas, am besten durch Ultrazinglas, ${ }^{32)}$ so verschwindet die

32) Ultrazin ist ein Derivat von Phenylglukosazon und nur für die sichtbaren Strablen durchlïssig. Vgl. T. Suzuki u. S. Sakurai, Bulletin of the Institute of Physical and Chemical Research, 1922, 4, 332 (Jap.). 
Fluoreszenz. Wenn auch das Tapetum des Selachiers bei Tageslicht schöne Irideszenz ähnlich wie beim Süugertapetum zeigt, fluoresziert es doch bei der Bestrahlung mit Ultraviolett nicht.

Die Fluoreszenzerscheinung kommt am schönsten heraus, wenn man das Licht der Nickelbogenlampe durch den oben besprochenen U-V-Filter auf das ausgetrocknete Tapetum fallen lüsst. Dieses starke Fluoreszenzlicht des Hunde- resp. Katzentapetums zeigt, mit einem Spektroskop betrachtet, ein kontinuierliches Spektrum, welches sich von 6500 bis 4500 A. E. (bei breiter Kollimatorspalte $7000--4150$ A. E.) erstreckt. Die Helligkeitsverteilung desselben verhält sich ähnlich wie die des gewöhnlichen Sonnenspektrums und ist verschieden je nach dem Adaptationszustand des beobachtenden Auges. Beim helladaptierten Auge erscheint das Gebiet von 5800 A.E. und beim dunkeladaptierten das von 5400 A.E. maximal hell, und die Helligkeit nimmt nach beiden Enden des Spektrums allmählich ab. Das Helligkeitsmaximum ist beim Photogramm(Fig. 8) nach noch kurzwelligerer Seite verschoben und liegt in der Gegend 4800 A.E., welche dem Gebiete des Empfindlichkeitsmaximums der panchromatischen Emulsion ${ }^{23)}$ entspricht.

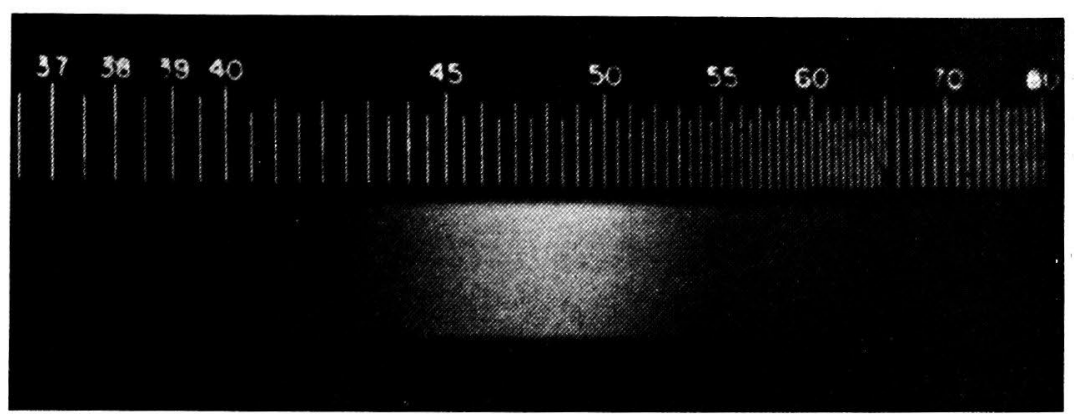

Fig. 8 .

Spektrogramm der Fluoreszenz des Katzentapetums (etwa 5,5 fach vergrös ert).

Da die schillernde Farbe des Tapetums mit dem Auslösen der Zelleinschlüsse durch Säuren oder Alkalien verschwindet, also jene von der Gegenwart der Zelleinschlüsse abhüngig ist, so untersuchte ich anch die Wirkung solcher Agentien auf die Fluoreszenz. Er ergab sich, dass beim mit $10 \% \mathrm{NaOH}$ oder $20 \% \mathrm{HCl}$ behandelten Tapetum die selbstleuchtende Eigenschaft verloren geht, während die Lösungen der Tapetumsubstanz deutlich fluoreszieren.

33) E. C. Bal y, Spectroscopy, 1927, Bd. 2., 362. 
Diese Fluoreszenz ist in Alkali stärker als in Süure, und beide geben ein ganz ähnliches Spektralbild wie das der Tapetumschicht (Fig. 9). Somit ist es erwiesen, dass die fluoreszierende Substanz des Tapetums bei Behandlung mit Säuren oder Alkalien auf die letzteren übergeht.

Die fluoreszierende Kraft der alkalischen resp. sauren Iösung der Tapetumsubstanz bleibt lange unverändert, übersteht die Siedehitze. Dagegen zeigt die entsprechende Auflösung aus den tapetumfreien Teilen der Chorioidea kaum Fluoreszenz.

Bei der Fluoreszenz handelt es sich um eine Verwandlung des ins Tapetum eindringenden kurzwelligen Lichtes in ein solches von anderer Farbe resp. Brechbarkeit. Wenn man nun das Auge eines Hundes, bei dem die Pigmententwicklung der Chorioidea schwach ist, nach der früher erwähnten Entfernung der Sklera in der hinteren Hemisphäre mit dem mit Blau-Uviolglas filtrierten Licht von vorn bestrahlt und von hinten betrachtet, so findet man, dass die Chorioidea in dem nicht tapezierten Teil wie eine halbdurchsichtige Membran relativ hell, in dem tapezierten dagegen als dunklerer Schatten erscheint. Es ist ziemlich merkwürdig, dass eine von vorn gesehene hell fluoreszierende Partie, von hinten betrachtet, dunkler erscheint. Dieselbe Erscheinung ist auch bei einer auf einen Objektträger flach aufgeklebten Chorioideaschicht sichtbar. Sie wird wenigstens zum Teil darauf beruhen, dass, abgesehen von der Reflexions- und Dickenverschiedenheit, die Tapetumschicht mehr Violett-und Ultraviolett-absorbiertals andere Chorioideateile, um dies in lïngerwellige Strahlen umzuwandeln.

Hier folgen Photogramme der Spektra von erregenden und emittierenden Lichtstrahlen.

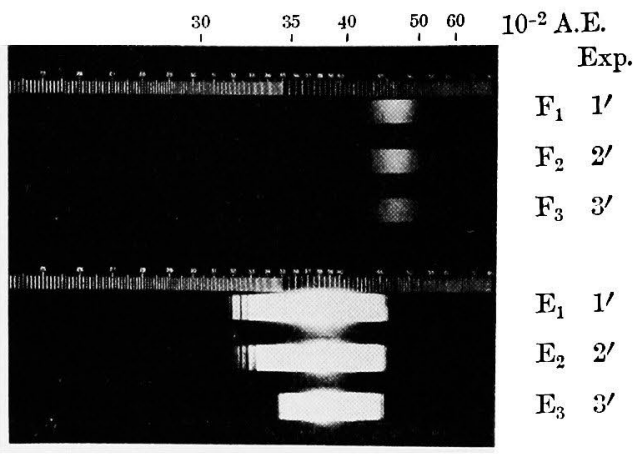

Fig. 9.

$\mathrm{E}_{1}-\mathrm{E}_{3}$ : Erregende Lichtstrahlen.

$\mathrm{E}_{1}: \mathrm{CuSO}_{4}(20 \%, \mathrm{smm}$ dick $)+$ Blau-Uviolglas.

$\mathrm{E}_{2}: \quad \quad \quad+\mathrm{U}-\mathrm{V}$-Glas.

$\mathrm{E}_{3}: \quad " \quad \quad$ Crookesglas.

$F_{1}-F_{3}$ : wit $E_{1}-E_{3}$ erregten Emissionsspektra d. Katzentapetums.

Kollimatorspalte ; bei $\mathrm{F}_{1}-\mathrm{F}_{3} 0,9 \mathrm{~mm}$, bei $\mathbf{E}_{1}-\mathbf{E}_{3}$ $0,046 \mathrm{~mm}$.

Man sieht in Fig. 9 oben die Bänder des blaugrünen Fluoreszenzlichtes des Tapetums, unten die Spektra des erregenden, durch verschiedene Filter 
gekommenen Nickelbogenlichtes, das sich weit über 4500 A. E. nach Ultraviolett erstreckt. Diese Beobachtung spricht für die Gültigkeit der Stokesschen Regel, da sie unmittelbar die Umwandlung violetter und ultravioletter Strahlen in gelbe, grüne und blaue zur Anschauung bringt.

Ferner wurde das Tapetum oder seine Alkalilösung (s. oben) mit verschieden breitem Ultraviolett bestrahlt, um das innerste Ende des ultravioletten Gebietes, welches die Fluoreszenz erregen kann, zu bestimmen. Beim Versuche diente ein mitUltrazinlösung (die zwar ultravioletteStrahlen zurückhält, aber bei starker Verdünnung mehr oder weniger durchlässt) gefüllter Glastrog als Filter. Dabei absorbiert das Glas natürlich die Strahlen von kürzer als 3000 A. E. gänzlich (Fig. 10), aber diese Absorption ist für diese Untersuchung von keinem Belang.

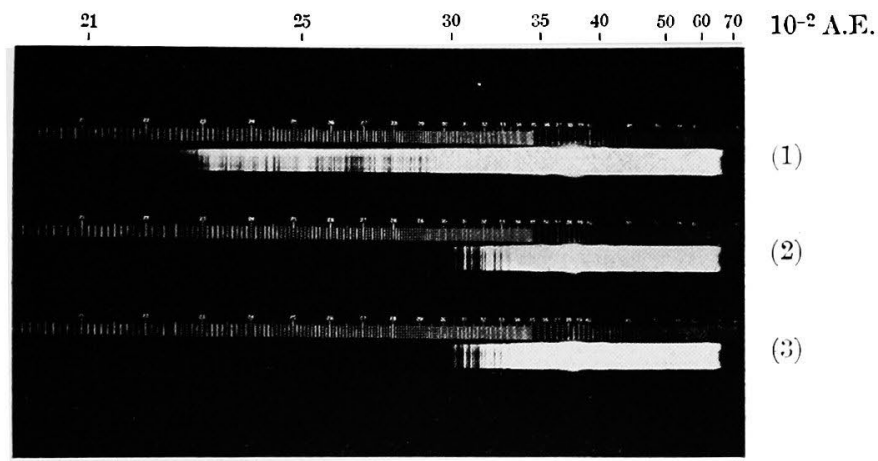

Fig. 10.

(1) Nickelbogenlicht.

(2) Absorptionsspektrum d. leeren Glastrogs.

(3) Absorptionsspektrum d. mit Wasser gefüllten Glastrogs.

Kollimatorspalte : 0,046 mm. Expositionsdauer : $30^{\prime \prime}$.

Wenn das Licht der Nickelbogenlampe durch die verschieden dünne Ultrazinlösung mit Quarzspektrograph auf die panchromatische Platte genommen wird, so tritt erst bei $\frac{1}{3} \times\left(\frac{1}{2}\right)^{4} \%$ Verdünnung eine Spur von Ultraviolett ca. $3100-3550$ A. E. auf, und mit weiterer Verdünnung nimmt die Breite des filtrierbaren ultravioletten Gebietes schnell zu (Fig. 11). Das durch diese verdünnte Ultrazinschicht kommende Ultraviolett kann die Fluoreszenz des Tapetums erregen.

Mit den im hiesigen Handel käuflichen Kobalt- und den Jenaer Blaugläsern, die als Filter für die Regulierung des hier in Frage stehenden Bezirkes von Ultraviolett gut anwendbar sind, führte ich noch mehrere Bestimmungen durch. Ein $3 \mathrm{~mm}$ dickes Kobaltglas lässt Rot (7200-6700 


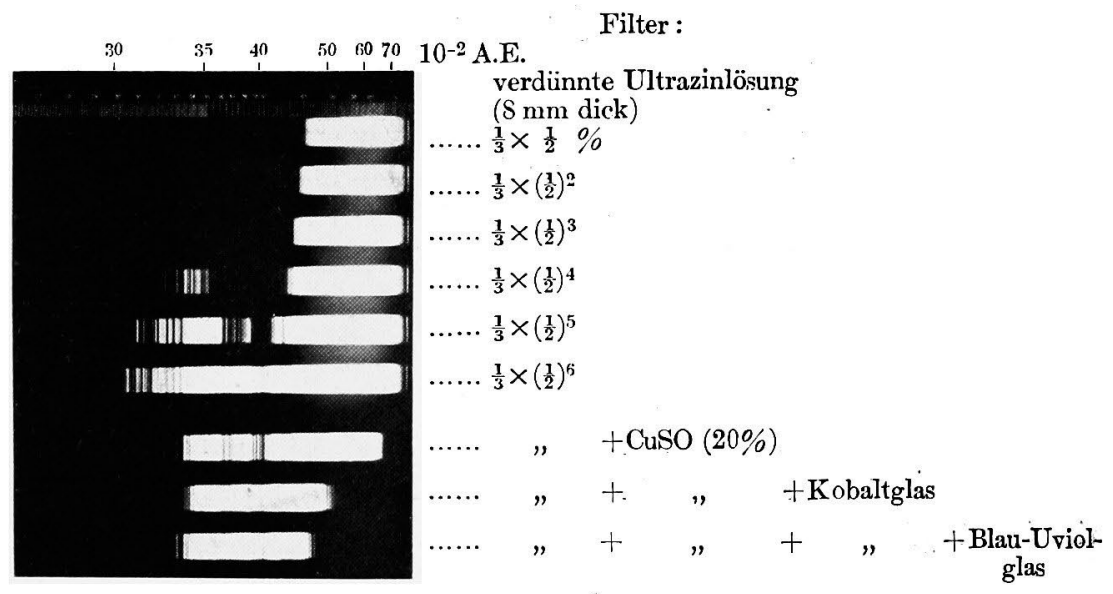

Fig. 11.

Kollimatorspalte : 0,092 mm. Expositionsdauer: $1^{\prime}$.

A.E.), Gelbgrün (5650-5450 A.E.) und von Blaugrün bis Ultraviolett (5200-3300 A.E.) durch, und zwar wird von beiden Enden des Spektrums um so mehr abgeschnitten, je mehr die Glasplatten geschichtet werden; durch die Schicht von 4 Kobaltgläsern passieren nur Strahlen von Wellenlänge 4500-3450 A. E. Beim 2,5 mm dicken Jenaer Blauglas erstreckt sich die Durchlässigkeit auf Gelb (5750-5450 A.E.) und von Grün bis Äusserstviolett (5300-3750 A.E.), durch 2 Glüser aber wird sie im Gebiete von Blaugrün bis $\ddot{A} u s s e r s t v i o l e t t ~(5100-3800$ A.E.) beschränkt, und das Licht auf einer Strecke 3950-3900 A.E. sogar abgehalten (vgl. Fig. 12).

Die genannten Durchlässigkeitsgrenzen, die spektroskopisch gemessen wurden, sind ziemlich rerschieden von den spektrographisch nachweisbaren, und vor allem kommt das Gebiet ron Rot und Gelb beim Spektrogramm (Fig. 12) in dieser Expositionszeit noch nicht zum Vorschein.

Wird das Tapetum durch die genannten Filter mit Nickelbogenlicht bestrahlt, so tritt die Fluoreszenz in verschiedener Intensität auf. In Fig. 12 sind die Spektra der erregenden Lichtstrahlen und die der dadurch erregten Fluoreszenz zusammengestellt.

Bei der Beleuchtung durch eine Blauglasplatte (Fig. 12, $\mathbf{E}_{7}$ ) bemerkte ich eine Spur von der Fluoreszenz des Tapetums. Diese war aber äussert schwach, so dass das Fluoreszenzlicht anch nach 5 minutiger Beleuchtung bei der ganz offenen Spalte des Kollimators auf panchromatische Platte gar nicht wirkte, und nach der Addition eines zweiten Blauglases (Fig. 12, $\mathbf{E}_{6}$ ) kaum mehr sichtbar wurde. Dies béruht nicht etwa auf der Stärkeab- 


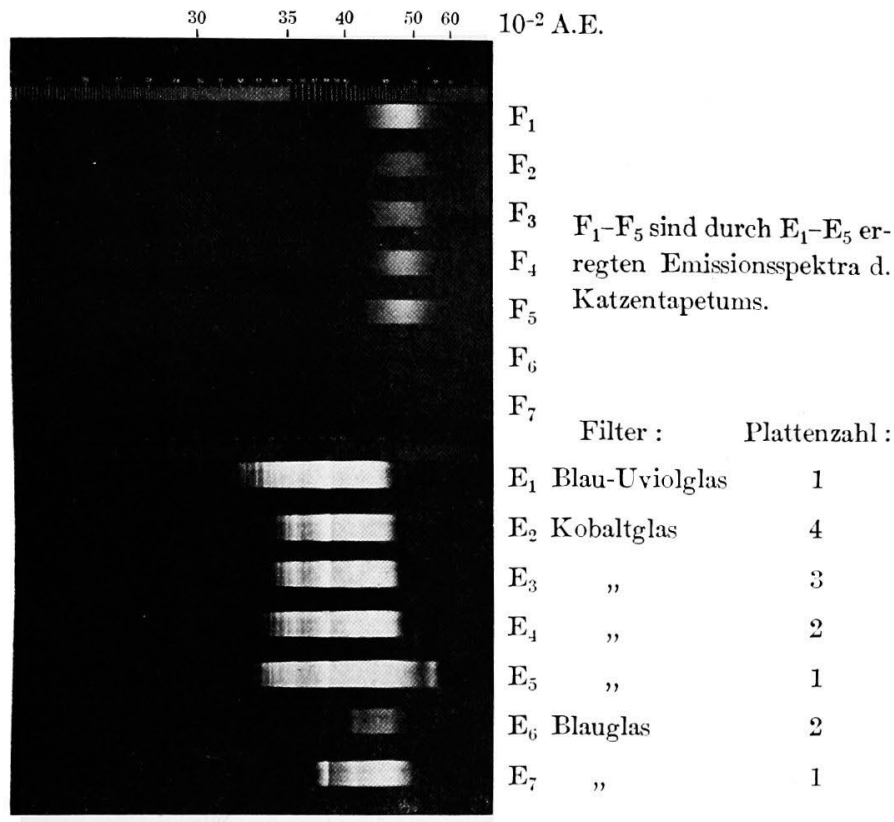

Fig. 12.

Lichtquelle : Nickelbogenlampe (đusserstrot mit $\mathrm{CuSO}_{4}$-Lösung absorbiert).

Kollimatorspalte :

$\mathrm{F}_{1}-\mathrm{F}_{5} \quad 0,9 \mathrm{~mm}$

$\mathrm{E}_{1}-\mathrm{E}_{\overline{7}} \quad 0,092$

Exposition :

$5^{\prime}$

2

nahme der erregenden Strahlen, sonderm auf der Absorption des Glases in der Wellenlänge 3880-3800 A. E., die fluoreszenzerregend wirkt, was durch die nächsten Experimente noch genauer klargestellt wird.

2. Herstellung eines Fluoreszenzschirmes aus der Tapetumschicht.

Um die Strahlengattungen, welche das Selbstleuchten des Tapetums verursachen, genau zu bestimmen, stellte ich eine Art von Fluoreszenzschirm in der Weise her, dass die frisch abpräparierte Chorioidea der Katzen-, Hunde- oder Rinderaugen auf einer photographischen Glasplatte ohne Gelatinhaut mit der tapezierten Fläche nach der Glasseite gcklebt, und nach dem Austrocknen die pigmentierte Aderhaut mit Messer vorsichtig abgeschabt und nur die Tapetumschicht zurückgelassen wurde. Auf diese Weise kann man einen auf einer Glasplatte aufgeklebten lediglich aus einer halbdurchsichtigen Tapetumschicht bestehenden Schirm bekommen. 
Wenn man diese Platte im Dunkelzimmer im Rahmen des Quarzspektrographs einsetzt und das Licht der Nickelbogenlampe durch die Spalte des Kollimators darauf einwirken lässt, so sieht man, dass mehrere fluoreszierende Linien, die der Spektrallinien des Nickels entsprechen, durch die halbdurchsichtige Tapetumschicht schimmern. Bringt man dabei das BlauUviolglas zwischen Lichtquelle und Spalte, so werden nun die Fluoreszenzlinien ganz auffallend, da die sichtbare Strecke des Spektrums grösstentcils abgeschnitten wird.

Bei der Bestrahlung ohne. Filter kann man mit blossem Auge auf diesem Schirm 4 Gruppen der leuchtenden Streifen, die in den bestimmten Gebieten des Spektrums lokalisieren, wahrnehmen und der Intensität nach in folgender Reihe aufzählen : 3950-3800 A.E., 3700-3400 A.E., 25402500 A.E. und 4250-4100 A.E., und zwar istunter diesen fluoreszierenden Gruppen die erste auf den ersten Blick deutlich bemerkbar, aber die letzte so minimal, dass sie erst nach maximaler Dunkeladaptation ganz schwach in die Augen fällt. Wenn man hinter dem leuchtenden 'Tapetumschirm eine panchromatische Platte einsetzt, so können die Fluoreszenzstreifen samt dem erregenden Spektrum photographiert werden (vgl. Fig. 13 (a). In Fig. 13 (b) ist ein Spektrogramm gesehen, auf dem auch die Strahlengattungen, die die Fluoreszenz des Tapetums stark erregen, durch Vergleichen der Fluoreszenz- und Spektrallinien ersichtlich sind. Aber die schwächst fluoreszierende Strecke kommt darauf nicht zum deutlichen Vorschein.

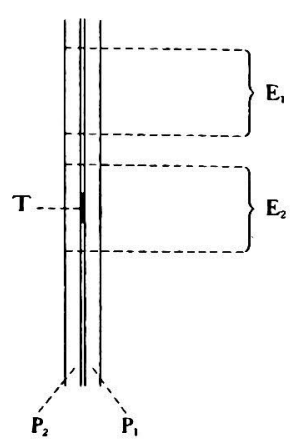

Fig. 13 (a).

Schema der Schichtungsweise der Platten beim Photographieren. $\quad P_{1}$ : Prïparatglasplatte. $P_{2}$ : Panchromatische Platte. T: Tapetumschicht.

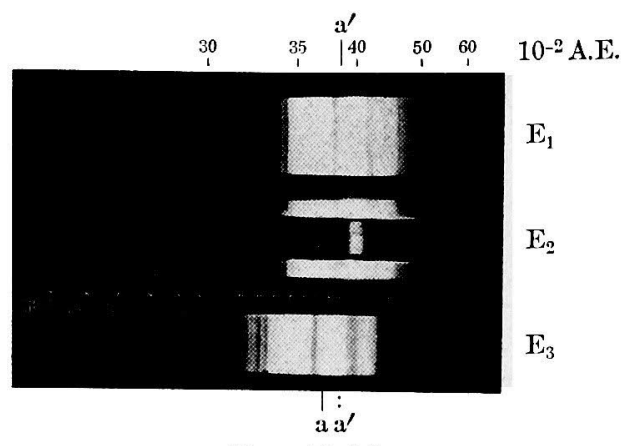

Fig. 13 (b).

Spektra des erregenden Lichtes und Fluoreszenzstreifen der Tapetunschicht (auf $\mathrm{P}_{2}$ photographiert). $\quad E_{1}$ : Spektrum des durch $P_{1}$ gekommenen Lichtes. $\mathrm{E}_{2}$ : Spektrum des durch $\mathrm{P}_{1}$ u. $\mathrm{T}$ (in der Mitte) gekommenen Lichtes. $\mathbf{E}_{3}$ : Spektrum des ohne $P_{1}$ u. $T$ direkt aufgenommenen Lichtes. Die Verschiebung der Banden $E_{1}$ und $\mathrm{E}_{2}$ von der $\mathrm{E}_{3}$ (um den Betrag $\mathrm{a}-\mathbf{a}^{\prime}$ ) beruht auf der Vorschaltung der Glasplatte $\mathrm{P}_{1}$, die zur Richtung des dispergierten Strahlenganges schief steht. 
Hieraus geht hervor, dass die Fluoreszenz des Tapetums auch bei sichtbarem Violett erregbar ist.

3. Absorptionsvermögen des Vollauges im Ultraviolett und die Fluoreszenz des Tapetums.

Die Ultravioletta bsorption von brechenlen Medien res Auges beim Tier oder Menschen wurde seit Brücke ${ }^{\text {st) }}$ von mehreren Forschern untersucht, und über die der sïmtlichen Augenmedien resp. des Vollanges-Cornea, Kanmerwasser, Kristallinse und Glaskörper-bei tapezierten Augen liegen die Arbeiten von Br ücke ${ }^{3 i j}$ (beim Rind), F. Schanz und K. Stockha u$\operatorname{sen}^{3 i)}$ (beim Kalb), Y. Shoji ${ }^{i i)}$ (bei Rind und Pferd) unl S. Russ $s^{35 j}$ vor. Vor allem hat Y. Shoji cine ganz genaue spektrographische Bestimmung ausgeführt und auch die Absorption der Netzhautschicht gemessen. Ferner fand Russ, dass das Auge der im Dämmer tätigen Tiere, z. B. Tiger, Katzen, Bären, Iöwen und Eulen, ultraviolette Strahlen gut durchlïsst, und sprach die Termutung aus, dass von solchen Tieren das die Retina erreichende Ultraviolett zur Tätigkeit in der Nacht benutzt werden könne. Aber auf das Wie ging er nicht ein. Nun sind die oberen Ergebnisse der Absorptionsmessung des tapezierten Auges im kurzwclligen Teil des Spektrums sehr interessant, da das Tapetum bei der Beleuchtung mit diesem Spektralgebiet stark fluoresziert, was aber bisher von niemand berücksichtigt worden ist.

Zur Bcobachtung bohrte ich, wie Shoji:i und Russ, am hinteren $\mathrm{Pol}$ des frisch enukleierten Auges ein kleines Fenster durch; wemn man nach der Durchbohrung der Sklera dic Chorioilea mit stumpfer Nadel vorsichtig ablöst, so klafft die kleine Wunde der Chorioidea von selbst; dabei muss die Retina frei von Schädigung sein. Das lenster wird mit einem Deckglas bedeckt, worauf dieses ganze Präparat von vorn (mit Cornea nach der Lichtseite) mit dem Nickelbogenlicht bestrahlt und von hinten spektrographiert wird.

Das gewöhnliche Glas hält zwar das Cltariolett in weitem Mase zurick, duch liisst die von $\mathrm{nir}$ gebrauchte diinne Deckglasplatte $(0,15 \mathrm{~mm}$ dick $)$ Strahlen bis $2750 \mathrm{~A}$. F. gut und hinunter bis 2700 A.F. schwach durch (Fig. 14).

34) E. Briicke, Arch. f. Anat. u. Thysiol, 1945, 262.

35) Derselbe, Ebenda, 1846, 579.

:6) F. Schanz u. D. Stockhausen, Arch. f. Ophthulmol, 150!, 69, 472.

37) Y. Shoji, Mitteil. ans d. ned. Fakult. Kaicerl. Cnivers. Toliyo, 1520, 29, 61.

3s) S. Russ, Nature, 1925, 16, 894.

39) Y.Shoji(3), 95-96. 


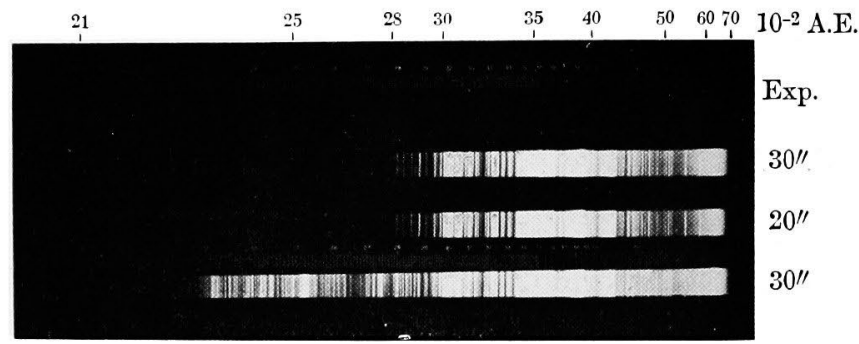

Fig. 14.

Zwei Absorptionspektra des Deckglases und ein Nickelbogenspektrum. Kollimatorspalte : $0,046 \mathrm{~mm}$.

Uber die mikroskopisch gemessenen Absorptionsgrenzen der verschiedenen Tieraugen gibt die folgende Tabelle Aufschluss (vgl. auch Fig. 15).

\begin{tabular}{ll} 
Versuchstier & \multicolumn{1}{c}{$\begin{array}{c}\text { Absorptionsgrenze } \\
\text { des Vollauges }\end{array}$} \\
Hund & 3220 A. E. \\
Katze & 3225 \\
Rind & 3355 \\
Pferd & 3710 \\
Ziege & 3525 (3700-3525 A. E. nur \\
& minimal durchlïssig)
\end{tabular}

\section{Anmerkung}

Lichtquelle :

Nickelbogenlicht, Konst. Str. 110 V. $5 \mathrm{Amp}$.

Kollimatorspalte : $0,092 \mathrm{~mm}$ breit, $\mathrm{Ab}-$ stand des Prüparates von der Lichtquelle: $50 \mathrm{~cm}$.

Daraus ist ersichtlich, dass das Ultraviolett, welches die Fluoreszenz des 'Tapetums erregt, durch sämtliche Augenmedien hindurch die Tapetumschicht im genügend breiten Umfang erreichen kann. In der Tat konnte ich nachweisen, dass die 'Tapetumschicht, sei es in frischem oder getrocknetem Zustande, deutliche Fluoreszenz zeigte, wenn sie mit dem durch das ganze hinten gefensterte Auge hindurch filtrierten Ultraviolett, das aus dem durch Blau-Uviolglas filtrierten Nickelbogenlicht stammt, bestrahlt wurde.

Daher lässt es sich vermuten, dass die Bedeutung des Tapetums nicht nur in der auf der Lichtreflexion beruhenden Reizverstärkung, sondern auch in der Ausnutzung der gewöhnlich schwer sichtbaren kurzwelligen Strahlen durch die oben besprochene Strahlenumwandlung (Hess) liegt, vorausgesetzt, dass auch für die genannten Süugetiere das für den Menschen sichtbare Spektralgebiet allein oder wenigstens besser als das andere sichtbar ist, da sonst eine Strahlenumwandlung unnötig oder gar unzweckmüssig sein muss. 


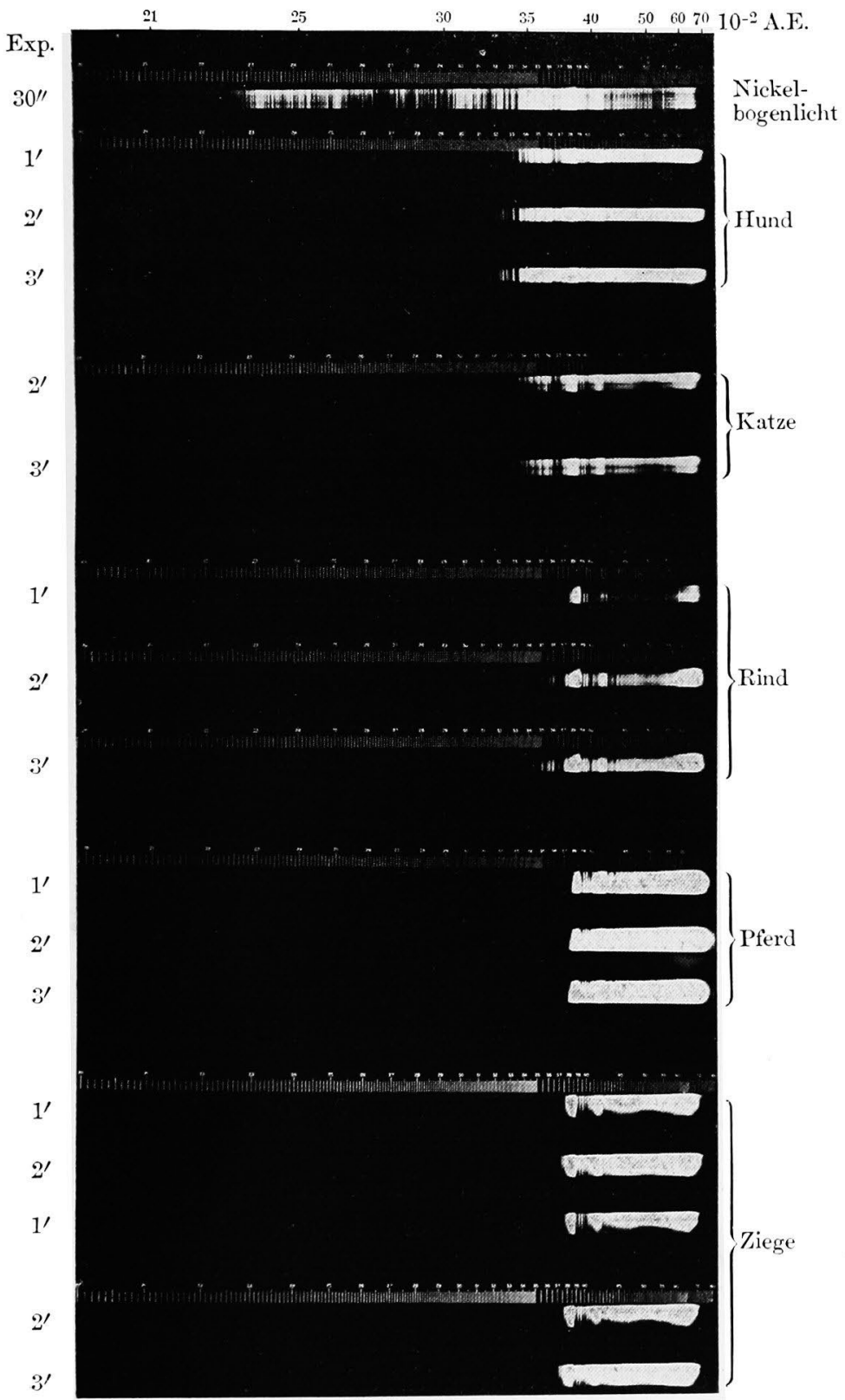

Fig. 15 .

Absorptionsspektra des Vollauges von verschiedenen Siugetieren (Kollimatorspalte : $0,046 \mathrm{~mm}$ ). 


\section{Zusammenfassung.}

(1) Das Netzhautpigmentepithel der meisten tapezierten Säugetieraugen ist in der mittleren Partie des Angenhintergrundes pigmentlos und nur beim Pferde stets, wenn auch ganz spärlich pigmenthaltig.

(2) Bei Rinder-, Pferde-, Ziegen- und Katzentapetum verschwindet der Farbenglanz mit dem Austrocknen, so dass dasselbe nicht vom übrigen Chorioideateil unterscheidbar wird, während es beim Hunde grauweisslich und deutlich erkennbar bleibt. Beim Befeuchten ausgetrockneterPräparate von den genannten Tieren erscheint der Farbenglanz sogleich wieder meist in tiefer blïulichem Ton.

(3) In Säuren kehrt die Farbe des Rindertapetums nach einmaligem Verschwinden in einer verdunkelten Nunance wieder zurück, wird dagegen in Alkalien allmählich grauweisslich und glanzlos. Beim Pferdetapetum tritt beim Zusatz von Alkalien nach einmaligem Verschwinden ein dunkelgräner Farbenglanz auf. Katzen- und Hundetapetum verlieren ihre Farbe sowohl in Säuren als auch in Alkalien gänzlich, und nach dem Austrocknen gewinnen die so behandelten Präparate auch in Wasser oder Ringerlösung dieselbe nicht mehr wieder. Beim Auflösen der Tapetumsubstanz in Salzsüure wurde die Entwicklnng des Gases, das nach $\mathrm{P}$ ütter $\mathrm{CO}_{2}$ sein soll, nicht nachgewiesen.

(4) Die mikrokristallihnlichen Gebilde (0.4-0.6 $\mu$ dick) sind nicht nur in den Katzen-, wie bisher geglaubt, sondern auch in den frischen Hundetapetrimzellen nachweisbar. Diese Gebilde sind beim Hunde sehr schwach resistent gegen die Fixier- oder Aufbewahrflüssigkeiten (Formol oder Sublimat), und vielleicht war ihr Vorkommen auch im Hundetapetum bisher deshalb ïberschen, weil sie gewöhnlich in fixierten Präparaten untersucht wurden.

(5) Da das Tapetum fibrosum aus $0,4-0,6 \mu$ dicken parallel verlaufenden Fasern besteht und das Tapetum cellulosum in seinen Zellen feine Büschel von streifig angcordneten mikrokristallähnlichen Gebilden von ebensolcher Feinheit enthält, so liegt es nahe, den Farbenglanz des Säugetiertapetums als ein Interferenzphänomen durch die Gitterbeugung anzunehmen.

(6) Im Tapetum nengeborener Katzen und Hunde oder beim Fötus der Rinder ist diese feine Streifung nicht erkennbar. Erst mit voller Entwicklung der letzeteren beginnt es seinen charakteristischen Farbenglanz zu zeigen.

(7) Die Tapetumzellen des Selachiers (Cynias und Raja) sind mit 
feinen, kleiner als $1 / 2$ dicken, durchsichtigen Täfelchen crfüllt, und damit wird die Schillerfarbe nach dem Prinzip von Iichtinterferenz an dünnen Schichten verursacht. Die Tapetumsubstanz des Selachiers zeigt Guaninreaktion, während diese beim Säuger fehlt.

(8) Frisch abgeschälte Tapetumschicht zejgt sich unter dem Miliroskop in durchfallendem und auffallendem Licht komplementärfarbig. Sowohl die Zelleinschlüsse des T. cellulosum wie auch die Fibrillen des T. fibrosum haben doppeibrechende Eigenschaft und beim letzteren wechselt die Farbe mit der Drehung des Analysators.

(9) Die Fluoreszenz des Süucertapetums ist durch die folgenden 4 Strahlengruppen erregbar: Am stärksten bei $3950-3800$ A.E., schwiicher bei 3700-3400 A.E. und am schwächsten bei $2540-2500$ A.E. und $4250-$ 4100 A.E. Die alkalische Auflösung geschabter Tapetumsubstanz zeigt besonders starke Fluoreszenz, dagegen die Iösung aus den tapetfreien Teilen der Chorioidea kaum eine solehe.

(10) Wenn auch das Tapetum des Selachiers ähnlich wie das der Säugetiere schöne Schillerfarbe zeigrt, ist diese etwas anderer Natur, indem die Mikrokristalle desselben weder fluoreszierende noch doppelbrechende Eigenschaft besitzen.

(11) Das Fluorezenzlicht des 'Tapetums zeigt ein sich über 65004500 A.E. erstreckendes kontinuierliches Spektrum, dessen Helligkeitsmaximum, mit dem dunkeladaptierten Auge beobachtet, bei 5400 A.E. liegt und sich zu beiden Seiten allmählich abschwächt.

(12) Bei allen untersuchten Sïugetierangen lassen sämtliche optische Medien die die Fluoreszenz des Tapetums stark erregenden ultravioletten Strahlen gut durch, die erst auf dem letzteren in die sichtbaren umgcwandelt werden.

Zum Schluss sei es mir gestattet, meinem hochverehrten Lehree, IIerm Prof. Dr. T. Fujita, fur seine freundliche Anregung zur Auffihrung dieser Arbeit meinen herzlichsten Dank auszusprechen. 